\title{
Estudo retrospectivo da casuística de felinos domésticos no Hospital Veterinário da Universidade Federal de Minas Gerais, no período de 2005 a 2014
}

\author{
[Retrospective study of the case load of domestic cats at the Veterinary Hospital of the \\ Universidade Federal de Minas Gerais from 2005 to 2014] \\ P.A. Lima', J.M. Barbieri' ${ }^{2}$, R. Ecco ${ }^{2}$, R.M.C. Guedes ${ }^{2}$, R. Serakides ${ }^{2}$, N.M. Ocarino ${ }^{2}$, \\ F. Pierezan ${ }^{2}$, R.L. Santos ${ }^{2} *$ \\ ${ }^{1}$ Aluna de pós-graduação - Escola de Veterinária - Universidade Federal de Minas Gerais - Belo Horizonte, MG \\ ${ }^{2}$ Escola de Veterinária - Universidade Federal de Minas Gerais - Belo Horizonte, MG
}

\section{RESUMO}

O objetivo deste estudo foi caracterizar as doenças dos gatos domésticos provenientes dos casos de necropsia e histopatologia do Hospital Veterinário da Universidade Federal de Minas Gerais (HV/UFMG), de 2005 a 2014. Foram analisados 408 exames de necropsia e 197 de biópsias, segundo o sexo, a faixa etária e a raça. Os diagnósticos mais frequentes incluíram doenças infecciosas/inflamatórias ou parasitárias (22,5\%), agentes físicos $(18,1 \%)$, doenças proliferativas $(15,2 \%)$ e degenerativas $(13,5 \%)$. Politraumatismo $(10,8 \%)$ foi responsável pelo maior número de mortes em felinos, sendo as chances três vezes maiores em animais com até 24 meses de idade ( $\mathrm{P}=0,005$ OR 3,47 [IC 95\%: 1,40-8,57]). Neoplasias epiteliais corresponderam a 26 diagnósticos, sendo 20 (4,9\%) casos de malignidade. A ocorrência de carcinoma e seus subtipos foi 18 vezes maior em gatos idosos ( $\mathrm{P}<0,01$ OR 18,15 [IC 95\%: 7,41-44,45]). A insuficiência renal crônica foi mais frequente em gatos com mais de 120 meses $(\mathrm{P}=0,01)$. Machos apresentaram nove vezes mais chances de desenvolver doenças do trato urinário inferior quando comparados às fêmeas ( $\mathrm{P}=0,001$ OR 9,50 [IC 95\%: 2,78$32,48])$. Em relação às biópsias, animais adultos a idosos foram 10 vezes mais representados $(\mathrm{P}<0,001 \mathrm{OR} 10,8$ [IC 95\%: 3,22-36,79]).

Palavras-chave: levantamento, macroscopia, infecciosa, intoxicação, gatos

\begin{abstract}
The aim of this study was to characterize the diseases of domestic cats based on necropsy and histopathological examinations at the Veterinary Hospital of the Universidade Federal de Minas Gerais (HV/UFMG) from 2005 to 2014. A total of 408 necropsy and 197 biopsy samples were analyzed according to gender, age, and breed. The most frequent diagnoses included infectious/inflammatory or parasitic diseases (22.5\%), physical agents (18.1\%), proliferative (15.2\%), and degenerative (13.5\%) diseases. Polytrauma $(10.8 \%)$ was responsible for the highest number of feline deaths, with the odds three times higher in animals up to 24 months of age $(P=0.005$ OR 3.47 [95\% CI: 1.40-8.57]). Epithelial neoplasms corresponded to 26 diagnoses, with 20 (4.9\%) malignant cases. The occurrence of carcinoma and its subtypes was 18-fold higher in older cats $(P<0.01$ OR 18.15 [95\% CI: 7.41-44.45]). Chronic renal failure was more frequent in cats over 120 months $(P=0.01)$. Males were nine times more likely to develop lower urinary tract diseases when compared to females ( $P=0.001$ OR 9.50 [95\% CI: 2.78-32.48]). Regarding the biopsies, adult to elderly animals were ten times more represented ( $P<0.001$ OR 10.8 [95\% CI: 3.22-36.79]).
\end{abstract}

Keywords: survey, macroscopy, infectious, intoxication, cats

\section{INTRODUÇÃO}

O gato doméstico (Felis catus) é uma espécie que vem se destacando como animal de companhia, principalmente devido à sua

Recebido em 4 de março de 2017

Aceito em 17 de maio de 2018

*Autor para correspondência (corresponding author)

E-mail: rsantos@vet.ufmg.br habilidade de adaptação em ambientes pequenos. Segundo o IBGE (2013), a população de felinos domésticos no Brasil é de 22 milhões de animais. De acordo com as estimativas do Centro de Controle de Zoonoses, há 275.603 cães e 54.985 gatos em Belo Horizonte (CCZ-PBH, 2017). 
Em relação aos aspectos comportamentais da espécie, os gatos têm como peculiaridade se isolar do ambiente quando doentes. Em muitos casos, os proprietários não conseguem observar os sinais clínicos manifestados pelo animal e, frequentemente, encontram o animal morto (Marlet e Maiorka, 2010). Dessa forma, o exame post mortem tem sido cada vez mais solicitado por proprietários e clínicos para determinação da causa da morte, principalmente quando a suspeita é de intoxicação intencional ou doença infecciosa (Xavier et al., 2007; Siqueira et al., 2015). Em relação aos exames histopatológicos, provenientes de biópsias, a finalidade é a obtenção de um diagnóstico mais preciso que auxilie tanto na conduta terapêutica quanto no estabelecimento dos fatores prognósticos (Siqueira, 2011).

Os estudos retrospectivos em medicina veterinária são utilizados para avaliação de uma população em um determinado período de tempo. O levantamento da casuísta é baseado na seleção de casos diagnosticados em necropsia, exames histopatológicos associados com os dados epidemiológicos, clínicos, exames laboratoriais e de imagem. Em muitos casos, as informações são escassas ou ausentes, sendo a necropsia uma ferramenta diagnóstica importante para determinar a causa da morte do animal (Marlet e Maiorka, 2010).

O objetivo deste estudo foi caracterizar as doenças de gatos domésticos encaminhados ao Hospital Veterinário da Universidade Federal de Minas Gerais (HV/UFMG), diagnosticadas por meio de necropsia e exames histopatológicos, no período de janeiro de 2005 a dezembro de 2014.

\section{MATERIAL E MÉTODOS}

Foi realizado um levantamento do número de casos de necropsias e biópsias de felinos domésticos realizadas no período de janeiro de 2005 a dezembro de 2014. A casuística foi obtida de dados arquivados no programa de registro informatizado de relatórios do Setor de Patologia do Hospital Veterinário da UFMG. As informações foram agrupadas em banco de dados (necropsias e biópsias), com o número de registro dos animais, por data, informações dos animais (sexo, raça e faixa etária) e diagnósticos morfológicos. Alguns animais apresentaram mais de um processo patológico, sendo estabelecido apenas um diagnóstico definitivo.

As conclusões anatomopatológicas dos exames de necropsia foram agrupadas, de acordo com as características do processo patológico, em 10 grupos: infecciosas/inflamatórias ou parasitárias, agentes físicos (traumatismos ou obstruções), proliferativos (neoplásico ou não neoplásico), degenerativas, intoxicações ou toxi-infecções (agentes tóxicos exógenos ou toxinas bacterianas), iatrogênicos (trans ou pós-cirúrgicos), circulatórios, nutricionais, metabólicas ou endócrinas, alterações do desenvolvimento (congênitos, genéticos ou hereditários) e outros (não incluídos em nenhuma das categorias anteriores). Essa classificação também foi utilizada por Fighera et al. (2008), em estudo retrospectivo da causa mortis em cães. Casos em que não foi possível estabelecer um diagnóstico definitivo foram incluídos no grupo "inconclusivos". Os felinos foram agrupados, segundo a faixa etária, em três categorias: filhote a jovem (zero a 24 meses); adulto a maduro (25 a 120 meses) e idoso (>120 meses) (Vogt et al., 2010). Os exames histopatológicos foram separados em dois grupos: processos neoplásicos (tecido, origem celular e comportamento biológico) e processos não neoplásicos (doenças infecciosas e/ou inflamatórias ou proliferativas não neoplásicas). As neoplasias foram classificadas de acordo com o tecido enviado e subdivididas segundo a origem celular em: epiteliais, mesenquimais, células redondas e tumores melanocíticos (Meuten, 2002). Foram considerados os diagnósticos previamente estabelecidos, segundo os relatórios disponíveis no banco de dados.

Foram calculadas as frequências e testada a associação dos grupos de doenças e dos principais diagnósticos dos achados de necropsias em relação às variáveis sexo, faixa etária e raça pelo teste exato de Fisher, sendo o nível de significância adotado de $\mathrm{P} \leq 0,05$. Além disso, foi calculada a estimativa da força de associação por Odds Ratio ajustada (OR) e o intervalo de confiança de 95\% (IC 95\%) (Dohoo et al., 2003). Todos os cálculos relacionados ao teste exato de Fisher e Odds Ratio foram realizados com o auxílio do programa estatístico STATA $12 ®$ (Statacorp, EUA). 


\section{RESULTADOS E DISCUSSÃO}

Com base no arquivo de dados do HV/UFMG, foram obtidos 605 casos de felinos domésticos, correspondentes a 408 necropsias e 197 biópsias, no período entre janeiro de 2005 e dezembro de 2014. Do número total de necropsias, as fêmeas tiveram frequência de 49,26\%, e os machos de $47,79 \%$. Gatos sem raça definida (SRD) foram mais frequentes do que animais com raça definida (Siamês, Persa, Angorá, Himalaia, Bengal e Sphynx), representando $72,7 \%$ do número total de necropsias. Entre as raças, os siameses $(10,2 \%)$ e persas $(8,8 \%)$ foram mais frequentes. Os demais animais com raça definida representaram, juntos, apenas 12 registros. $\mathrm{O}$ número de felinos necropsiados segundo a faixa etária foi de $141(34,6 \%), 109(26,6 \%)$ e 36 $(8,7 \%)$, para filhote a jovem (zero a 24 meses), adulto a maduro (25 a 120 meses) e idoso (>120 meses), respectivamente. Animais sem informações de idade representaram 30,1\% (122 casos). A frequência do grupo de doenças em relação à faixa etária, ao sexo e à raça dos casos de necropsia está detalhada na Tab. 1.

Tabela 1. Frequência dos grupos de doenças por faixa etária, sexo e raça dos casos de necropsias de felinos domésticos realizadas pelo setor de patologia do HV-UFMG, de janeiro de 2005 a dezembro de 2014

\begin{tabular}{|c|c|c|c|c|c|c|c|c|}
\hline \multirow[b]{2}{*}{ Grupos de doenças } & \multicolumn{3}{|c|}{ Categoria de idade $(\%)^{* *}$} & \multicolumn{2}{|c|}{$\operatorname{Sexo}(\%)^{* *}$} & \multicolumn{2}{|c|}{$\operatorname{Raça~}(\%)^{* *}$} & \multirow[b]{2}{*}{ Total (\%) } \\
\hline & $\begin{array}{l}\text { Filhote a } \\
\text { jovem }\end{array}$ & $\begin{array}{l}\text { Adulto a } \\
\text { maduro }\end{array}$ & Idoso & Fêmea & Macho & Indefinida & $\begin{array}{l}\text { Definida } \\
* * *\end{array}$ & \\
\hline $\begin{array}{c}\text { Doenças } \\
\text { infecciosas/inflamatórias } \\
\text { ou parasitárias }\end{array}$ & $\begin{array}{c}20,8 \\
(30 / 144)^{\mathrm{a}}\end{array}$ & $\begin{array}{c}27,1 \\
(29 / 107)^{\mathrm{a}}\end{array}$ & $\begin{array}{c}8,3 \\
(3 / 36)^{b}\end{array}$ & $\begin{array}{c}19,4 \\
(39 / 201)\end{array}$ & $\begin{array}{c}24,6 \\
(48 / 195)\end{array}$ & $\begin{array}{c}24,1 \\
(72 / 299)\end{array}$ & $\begin{array}{c}18,0 \\
(16 / 89)\end{array}$ & $\begin{array}{c}22,5 \\
(92 / 408)\end{array}$ \\
\hline $\begin{array}{l}\text { Distúrbios causados por } \\
\text { agentes físicos }\end{array}$ & $\begin{array}{c}20,8 \\
(30 / 144)^{\mathrm{a}}\end{array}$ & $\begin{array}{c}14,0 \\
(15 / 107)^{\mathrm{a}}\end{array}$ & $\begin{array}{c}2,8 \\
(1 / 36)^{b}\end{array}$ & $\begin{array}{c}12,4 \\
(25 / 201)\end{array}$ & $\begin{array}{c}24,1 \\
(47 / 195)^{*}\end{array}$ & $\begin{array}{c}18,4 \\
(55 / 299)\end{array}$ & $\begin{array}{c}15,7 \\
(14 / 89) \\
\end{array}$ & $\begin{array}{c}18,1 \\
(74 / 408)\end{array}$ \\
\hline Doenças degenerativas & $\begin{array}{c}11,1 \\
(16 / 144)\end{array}$ & $\begin{array}{c}15,0 \\
(16 / 107) \\
\end{array}$ & $\begin{array}{c}25 \\
(9 / 36) \\
\end{array}$ & $\begin{array}{c}15,0 \\
(30 / 201)\end{array}$ & $\begin{array}{c}12,8 \\
(25 / 195) \\
\end{array}$ & $\begin{array}{c}11,4 \\
(34 / 299) \\
\end{array}$ & $\begin{array}{c}21,3 \\
(19 / 89)^{*}\end{array}$ & $\begin{array}{c}13,5 \\
(55 / 408)\end{array}$ \\
\hline $\begin{array}{l}\text { Intoxicações e toxi- } \\
\text { infecções }\end{array}$ & $\begin{array}{c}9,0 \\
(13 / 144)\end{array}$ & $\begin{array}{c}8,4 \\
(9 / 107)\end{array}$ & $\begin{array}{c}2,8 \\
(1 / 36)\end{array}$ & $\begin{array}{c}7,4 \\
(15 / 201)\end{array}$ & $\begin{array}{c}6,6 \\
(13 / 195)\end{array}$ & $\begin{array}{c}6,3 \\
(19 / 299)\end{array}$ & $\begin{array}{c}10,1 \\
(9 / 89)\end{array}$ & $\begin{array}{c}6,8 \\
(28 / 408)\end{array}$ \\
\hline Distúrbios iatrogênicos & $\begin{array}{c}10,4 \\
(15 / 144)^{\mathrm{b}}\end{array}$ & $\begin{array}{c}4,7 \\
(5 / 107)^{\mathrm{a}} \\
\end{array}$ & $\begin{array}{c}0,0 \\
(0 / 36)^{\mathrm{a}}\end{array}$ & $\begin{array}{c}8,4 \\
(17 / 201)^{*}\end{array}$ & $\begin{array}{c}3,1 \\
(6 / 195) \\
\end{array}$ & $\begin{array}{c}6,7 \\
(20 / 299) \\
\end{array}$ & $\begin{array}{c}3,4 \\
(3 / 89) \\
\end{array}$ & $\begin{array}{c}5,6 \\
(23 / 408) \\
\end{array}$ \\
\hline $\begin{array}{c}\text { Doenças do } \\
\text { desenvolvimento }\end{array}$ & $\begin{array}{c}1,4 \\
(2 / 144)\end{array}$ & $\begin{array}{c}0,9 \\
(1 / 107)\end{array}$ & $\begin{array}{c}0,0 \\
(0 / 36)\end{array}$ & $\begin{array}{c}0,5 \\
(1 / 201) \\
\end{array}$ & $\begin{array}{c}2,1 \\
(4 / 195)\end{array}$ & $\begin{array}{c}1,0 \\
(3 / 299)\end{array}$ & $\begin{array}{c}1,78 \\
(2 / 89)\end{array}$ & $\begin{array}{c}1,2 \\
(5 / 408)\end{array}$ \\
\hline Outros distúrbios & $\begin{array}{c}5,5 \\
(8 / 144) \\
\end{array}$ & $\begin{array}{c}1,9 \\
(2 / 107)\end{array}$ & $\begin{array}{c}0,0 \\
(0 / 36) \\
\end{array}$ & $\begin{array}{c}3,5 \\
(7 / 201) \\
\end{array}$ & $\begin{array}{c}2,1 \\
(4 / 195) \\
\end{array}$ & $\begin{array}{c}3,3 \\
(10 / 299) \\
\end{array}$ & $\begin{array}{c}1,1 \\
(1 / 89) \\
\end{array}$ & $\begin{array}{c}2,7 \\
(11 / 408)\end{array}$ \\
\hline Inconclusivos & $\begin{array}{c}4,1 \\
(6 / 144) \\
\end{array}$ & $\begin{array}{c}7,5 \\
(8 / 107) \\
\end{array}$ & $\begin{array}{c}0,0 \\
(0 / 36) \\
\end{array}$ & $\begin{array}{c}10,0 \\
(20 / 201)\end{array}$ & $\begin{array}{c}3,6 \\
(7 / 195) \\
\end{array}$ & $\begin{array}{c}6,3 \\
(19 / 299) \\
\end{array}$ & $\begin{array}{c}5,6 \\
(5 / 89) \\
\end{array}$ & $\begin{array}{c}6,7 \\
(27 / 408) \\
\end{array}$ \\
\hline Total $(\%)$ & $\begin{array}{c}35,3 \\
(144 / 408) \\
\end{array}$ & $\begin{array}{c}26,2 \\
(107 / 408) \\
\end{array}$ & $\begin{array}{c}8,8 \\
(36 / 408) \\
\end{array}$ & $\begin{array}{c}49,2 \\
(201 / 408) \\
\end{array}$ & $\begin{array}{c}47,8 \\
(195 / 408) \\
\end{array}$ & $\begin{array}{c}73,3 \\
(299 / 408) \\
\end{array}$ & $\begin{array}{c}21,8 \\
(89 / 408) \\
\end{array}$ & \\
\hline
\end{tabular}

*Indica diferença estatisticamente significativa nas comparações de sexo ou raça pelo teste exato de Fisher $(\mathrm{P}<0,05)$.

${ }^{a, b}$ Letras diferentes na mesma linha da categoria de idade indicam diferença estatisticamente significativa pelo teste exato de Fisher $(\mathrm{P}<0,05)$.

** Em 121 casos, não foram informadas as idades, em 12 casos não foi informado o sexo, e em 20 casos não foi informada a raça dos animais necropsiados.

***Siamês, Persa, Angorá, Himalaia, Bengal e Sphynx.

Os maiores números de diagnósticos entre as categorias foram provenientes, respectivamente, de causas de origem infecciosa/inflamatória ou parasitária $(22,5 \%)$, de agentes físicos $(18,1 \%)$, proliferativa $(15,2 \%)$ e degenerativa $(13,5 \%)$.
Em relação à faixa etária, animais até 120 meses de idade tiveram três vezes mais doenças infecciosas do que gatos idosos $(\mathrm{P}=0,025$ OR 3,38 [IC 95\%: 1,06-10,73]). Felinos da faixa etária filhote a jovem foram duas vezes mais acometidos por distúrbios físicos quando comparados a animais das demais faixas etárias 
( $\mathrm{P}=0,019$ OR 2,08 [IC 95\%: 1,09-3,99]). Entre as doenças proliferativas, gatos idosos apresentaram nove vezes mais chances de desenvolver uma doença neoplásica quando comparados a felinos das demais idades $(\mathrm{P}<0,001$ OR 9,67 [IC 95\%: 4,89-19,10]). Gatos com até 24 meses de idade foram três vezes mais acometidos por distúrbios iatrogênicos em relação às demais categorias de idade ( $\mathrm{P}=0,018$ OR 3,280[IC 95\%: 1,18-8,67]). Em machos, a morte ocasionada por agentes físicos foi duas vezes maior do que em fêmeas ( $\mathrm{P}=0,001$ OR 2,23 [IC 95\%: 1,32-3,77]). A Tab. 2 sumariza a frequência dos principais diagnósticos em relação à faixa etária, ao sexo e à raça dos casos de necropsia.

Tabela 2. Frequência dos principais diagnósticos por faixa etária, sexo e raça dos casos de necropsias de felinos domésticos do HV-UFMG, de janeiro de 2005 a dezembro de 2014

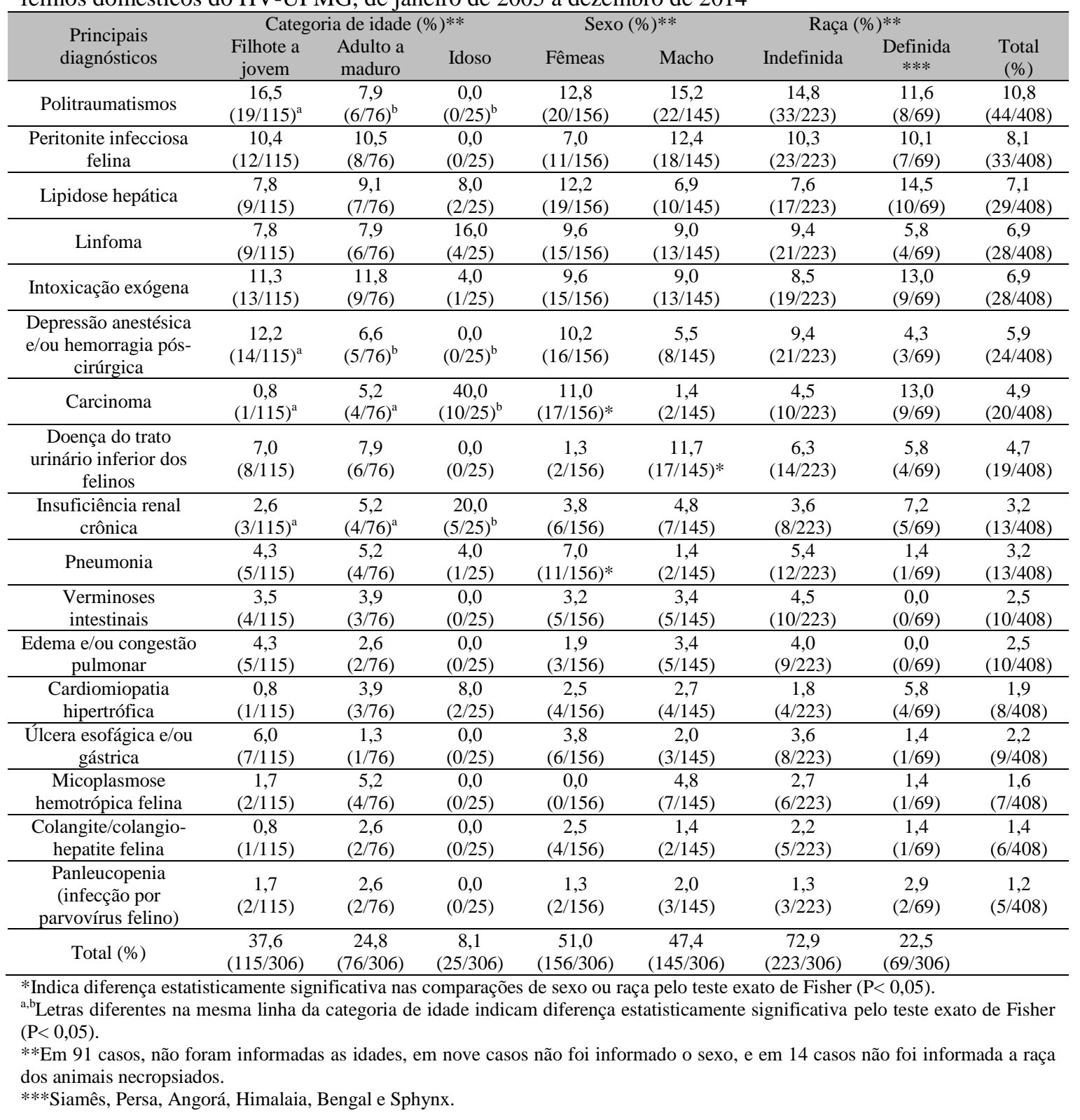


Segundo a Tab. 2, os politraumatismos $(10,8 \%)$ foram responsáveis pelo maior número de mortes em gatos durante o período de estudo. Nesse grupo, foram incluídos traumatismos decorrentes de mordedura de cães, atropelamentos ou trauma de origem desconhecida. As principais lesões encontradas foram fraturas ósseas múltiplas, perfurações da pleura parietal e visceral, hérnia diafragmática adquirida (Fig. 1A), rupturas de vísceras da cavidade abdominal e torácica com consequente extravasamento de sangue para cavidades corpóreas (hemotórax e hemoperitônio).

Animais com até 24 meses de idade apresentaram três vezes mais chances de desenvolver politraumatismos quando comparado aos das demais faixas etárias $(\mathrm{P}=0,005$ OR 3,47 [IC 95\%: 1,40-8,57]). Esse resultado está possivelmente relacionado com a fase de maturidade sexual dos animais, que leva ao acesso à rua e predispõe a traumatismos acidentais ou intencionais (Marlet e Maiorka, 2010). Em estudo retrospectivo em gatos que tiveram morte súbita, a causa principal de morte também foi atribuída a trauma (Olsen e Allen, 2001). Os resultados encontrados estão de acordo com os achados de Siqueira et al. (2015), nos quais observaram maior frequência de traumas intencionais na faixa etária de sete meses a dois anos de idade.

A ocorrência de doenças degenerativas em felinos com raça definida foi duas vezes maior do que em gatos sem raça definida $(\mathrm{P}=0,015 \mathrm{OR}$ 2,11[IC 95\%: 1,14-3,89]). O maior número de diagnósticos incluídos na categoria doenças degenerativas foi concentrado em lesões hepáticas (lipidose), representando 29 casos. Em 24 relatórios de necropsias, a morte por depressão anestésica e hemorragia pós-cirúrgica nos casos de esterilização eletiva foi mais frequente em felinos com até 24 meses de idade $(\mathrm{P}=0,028)$. Animais nessa faixa etária apresentaram ocorrência três vezes maior do que gatos das demais categorias de idade (OR 2,97 [IC 95\%: 1,08-8,14]).

O linfoma foi o tumor mais frequente, representando $6,9 \%$ do número total de diagnósticos de necropsia (Fig. 1B). Em felinos de regiões endêmicas, aproximadamente $70 \%$ dos casos de linfoma são atribuídos à infecção pelo vírus da leucemia felina $(\mathrm{FeLV}) \mathrm{e}$, recentemente, também pelo vírus da imunodeficiência felina (FIV) (Fighera e Graça, 2016). Embora os linfomas sejam descritos como as neoplasias mais frequentes em gatos com mais de 12 meses (Schmidt et al., 2010; Schweigert et al., 2011), não foi observada associação estatisticamente significativa entre a ocorrência dessa neoplasia e a faixa etária dos animais deste estudo. No presente estudo, foram diagnosticados histologicamente um linfoma cutâneo e oito casos de linfoma no trato digestório, que foram classificados como linfoblástico $(n=3)$, de células grandes $(n=1)$, difuso $(n=1)$ e não classificados $(n=3)$. Com base nos achados de necropsia, foram diagnosticados 28 casos de linfoma, com a seguinte classificação: multicêntrico $(n=12)$, tímico/mediastínico $(\mathrm{n}=10)$, intestinal $(\mathrm{n}=3)$, cutâneo $(n=1)$ e não classificados $(n=2)$.

Neoplasias de origem epitelial corresponderam a 26 diagnósticos, sendo $20 \quad(4,9 \%)$ amostras classificadas como malignas. A ocorrência de carcinoma e seus subtipos foi 18 vezes maior em gatos idosos ( $\mathrm{P}<0,01$ OR 18,15 [IC 95\%: 7,41$44,45]$ ). Nessa faixa etária, foram diagnosticados 10 casos, sendo sete fêmeas com carcinoma mamário. Fêmeas apresentaram nove vezes mais chances do desenvolvimento de carcinomas do que os machos ( $\mathrm{P}<0,001$ OR 8,91 [IC 95\%: 2,57$30,84])$. Os casos de carcinoma mamário foram classificados como: tubular $(\mathrm{n}=10)$, cribriforme $(\mathrm{n}=6)$, sólido $(\mathrm{n}=6)$, túbulo-papilar $(\mathrm{n}=5)$, papilar $(\mathrm{n}=2)$, in situ $(\mathrm{n}=2)$ e anaplásico $(\mathrm{n}=1)$.

A insuficiência renal crônica foi cinco vezes mais frequente em gatos com mais de 120 meses de idade ( $\mathrm{P}=0,010$ OR 5,44 [IC 95\%: 1,8216,23]). A causa mortis ocasionada por doenças do trato urinário inferior de felinos (DTUIF) foi nove vezes maior no sexo masculino quando comparado ao feminino ( $\mathrm{P}=0,001$ OR 9,50 [IC 95\%: 2,78-32,48]). O diagnóstico de úlcera esofágica e/ou gástrica (Fig. 1C) foi mais frequente em gatos da faixa etária filhote a jovem $(\mathrm{P}=0,034)$. Animais com até 24 meses apresentaram sete vezes mais chances de serem acometidos do que gatos das demais idades (OR 7,25 [IC 95\%: 1,17-44,64]). À necropsia, os rins com fibrose difusa apresentavam-se pálidos, irregulares, diminuídos de tamanho e com formação de cistos de retenção (Fig. 1D). Dentre as alterações extrarrenais de uremia, foi possível observar lesões ulcerativas na cavidade oral, 
caracterizadas por formação de material mucoide castanho de odor fétido, aderente às mucosas ulceradas ou erodidas (Fig. 1E). As lesões primárias nos casos de insuficiência renal crônica foram as seguintes: glomerulopatias $(n=3)$, fibrose e nefrocalcinose $(n=1)$, hipoplasia peniana $(n=1)$ e causas não definidas $(n=7)$.

Nos casos de peritonite infecciosa felina (PIF), não houve diferença significativa $(\mathrm{P}>0,05) \mathrm{em}$ relação à idade, ao sexo ou à raça dos animais. Gatos de todas as idades podem ser acometidos, no entanto parece haver predileção pela faixa etária de seis e 24 meses e acima de 13 anos (Guedes et al., 2016). Em estudo retrospectivo realizado na Inglaterra, a principal causa mortis de gatos filhotes foi decorrente de patologias infecciosas, sobretudo de origem viral (Cave et al., 2002). Macroscopicamente, a principal alteração encontrada nos casos de PIF efusiva foi caracterizada por um processo exsudativo intenso, com acúmulo de líquido amarelado, translúcido, com focos de fibrina nas cavidades abdominal (Fig. 1F) ou torácica. Na forma não efusiva ou seca, foram observadas lesões nodulares (piogranulomas) em diversos órgãos, principalmente rins, linfonodos e fígado.

A maioria dos casos de colangite diagnosticados foram caracterizados morfologicamente por colangite linfoplasmocitária associada ao parasitismo por Platynosomum fastosum $(\mathrm{n}=5)$, tendo sido diagnosticado um caso de colangiohepatite piogranulomatosa. Já as helmintoses gastrointestinais foram associadas a Toxocara cati $(\mathrm{n}=2)$, Dipylidium sp. $(\mathrm{n}=1)$, embora a maioria dos casos não tenha tido a identificação do parasito $(n=7)$.

Em 28 relatórios com diagnóstico sugestivo de intoxicação exógena aguda, o histórico clínico geralmente era de morte súbita. As lesões macroscópicas encontradas foram congestão, edema e hemorragia em diversos órgãos, como pulmões, fígado e rins. Em 11 casos, foi possível encontrar, em meio ao conteúdo estomacal, grânulos pretos, compatíveis com produto à base de Aldicarb. Lesões semelhantes também foram relatadas por outros autores em estudos retrospectivos (Xavier et al., 2007; Siqueira et al., 2015).

Do número total de biópsias, as fêmeas representaram $56,85 \%$, e os machos $43,15 \%$.
Gatos sem raça definida (SRD) representaram $66 \%$ do número total de biópsias, seguidos das raças Siamês $(9,64 \%)$ e Persa $(8,63 \%)$. As raças Himalaia e Angorá representaram, juntas, apenas cinco registros. Dos 197 exames histopatológicos, a faixa etária classificada como adultos a maduros foi a mais frequente $(43,15 \%)$. A categoria senil a idosos representou $24,87 \%$ do número total de exames, e a menos frequente foi incluída no grupo filhotes a jovens $(7,61 \%)$.

As neoplasias representaram $145(73,6 \%)$ das 197 biópsias recebidas durante o período do estudo. Os processos classificados como não neoplásicos (doenças inflamatórias e/ou infecciosas e proliferativas não neoplásicas) totalizaram $52(26,4 \%)$ casos. A distribuição dos exames histopatológicos, por faixa etária, está detalhada na Tab. 3 .

De acordo com os dados da Tab. 3, felinos adultos a idosos tiveram 10 vezes mais chances de desenvolver doenças neoplásicas quando comparados a animais da faixa etária filhotes a jovens ( $\mathrm{P}<0,001$ OR 10,8 [IC 95\%: 3,22-36,79]). Em relação ao tecido encaminhado para análise histopatológica, as neoplasias foram provenientes dos seguintes sistemas: tegumentar $(40,61 \%)$ e reprodutor feminino $(17,7 \%)$. Os demais sistemas (urinário, respiratório, musculoesquelético, hematopoético, nervoso, ouvido e bulbo ocular e anexos) representaram $8,63 \%$ dos diagnósticos. Na Tab. 4, está representado o número de diagnósticos das principais neoplasias, segundo os tecidos recebidos para exame.

Tumores epiteliais representaram 35,5\% $(n=70)$ dos diagnósticos de neoplasia, sendo 68 com características de malignidade. Dados semelhantes também foram descritos por outros autores (Schmidt et al., 2010; Schweigert et al., 2011). Na análise dos tumores de mama, houve predomínio dos carcinomas e seus subtipos, com 35 diagnósticos, o que mostra que, em gatas, as neoplasias mamárias geralmente apresentam comportamento maligno. Togni et al. (2013), em um estudo sobre tumores de mama em gatas, concluíram que os carcinomas mamários são diagnosticados com maior frequência, sendo o padrão túbulo-papilar e cribriforme os subtipos mais comuns. Entre as neoplasias de origem mesenquimal, o maior número foi de sarcomas cutâneos, com maior frequência o fibrossarcoma. 
Em 12 casos, o desenvolvimento do tumor estava relacionado ao local de aplicação de vacina (Couto et al., 2002). Semelhante ao encontrado nos exames de necropsia, a maior parte dos diagnósticos de neoplasias de células redondas foi de linfoma e seus subtipos, principalmente de origem no trato digestório. A classificação dos tipos de linfomas foi baseada nas características celulares ou na localização anatômica.
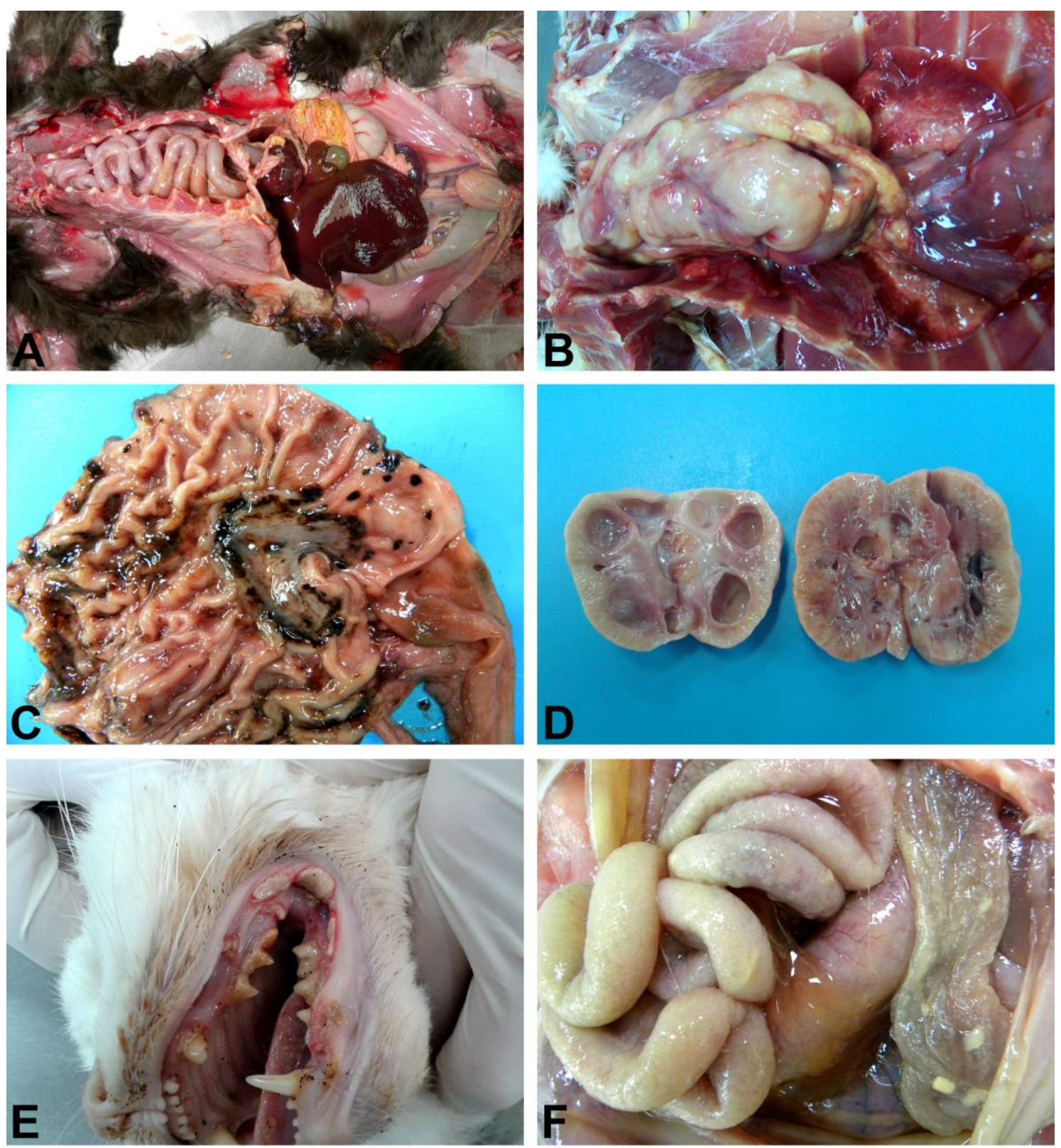

Figura 1. Felino. (A) Hérnia diafragmática, caracterizada por ruptura do diafragma e localização ectópica das vísceras abdominais dentro da cavidade torácica. (B) Linfoma mediastínico, tecido neoformado, irregular e esbranquiçado que obliterava o mediastino. (C) Úlcera gástrica, múltiplas úlceras associadas a hemorragias na mucosa gástrica. (D) Rins com fibrose intensa e várias estruturas císticas no córtex interno e na região medular. (E) Estomatite ulcerativa e necrosante, caracterizada por material mucoide castanho aderido às mucosas ulceradas ou erodidas. (F) Forma efusiva da peritonite infecciosa felina, serosa irregular e espessada com deposição de exsudato amarelado. 


\section{Lima et al.}

Tabela 3. Frequência dos grupos de doenças, por faixa etária, dos casos de biópsias de felinos domésticos analisadas pelo setor de patologia do HV-UFMG, de janeiro de 2005 a dezembro de 2014

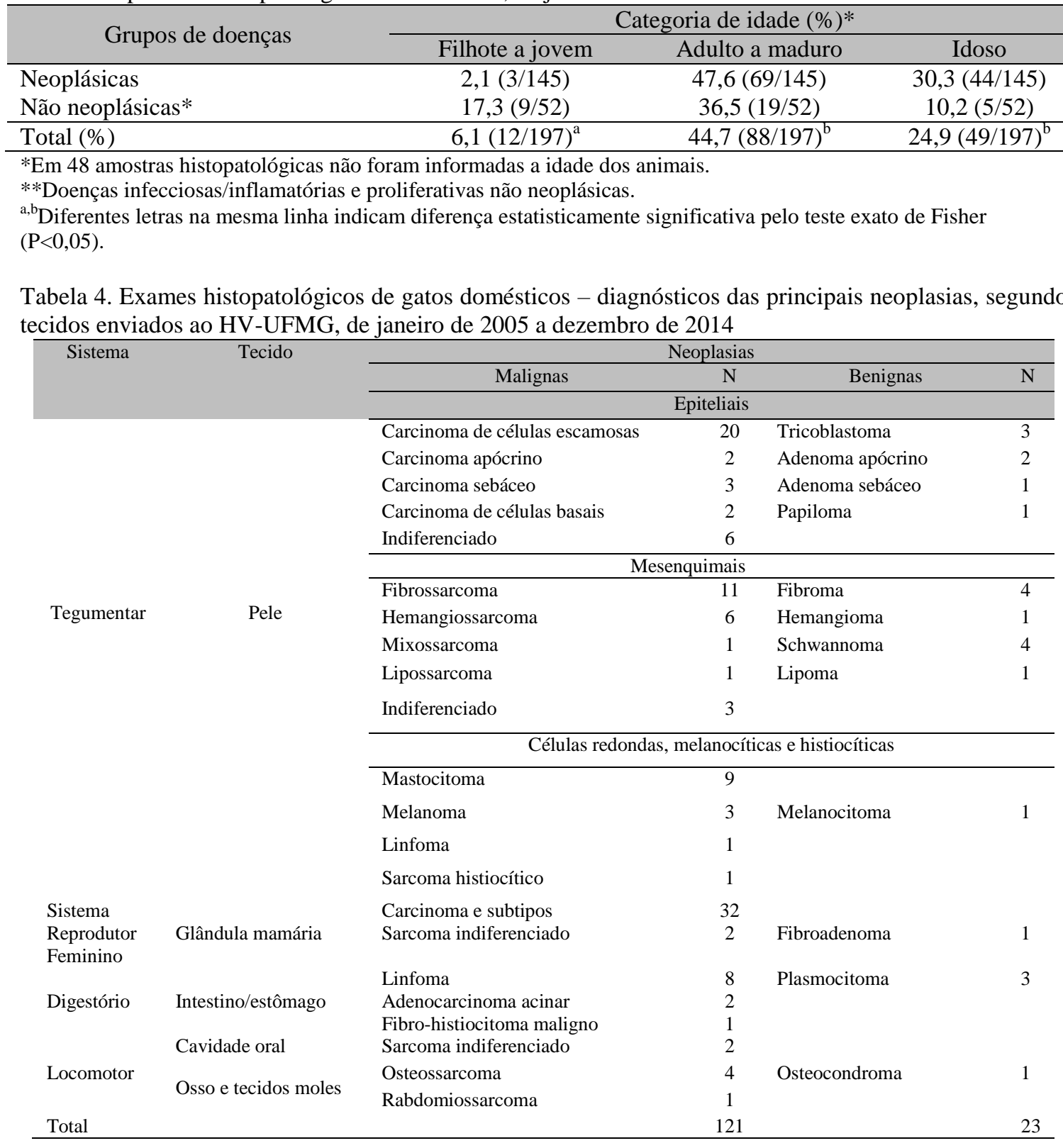

Em relação aos processos não neoplásicos, o maior número de amostras foi proveniente do sistema tegumentar, com 19 diagnósticos, principalmente processos inflamatórios crônicos. Dermatites piogranulomatosas, destacando-se a esporotricose $(n=8)$, foram as mais diagnosticadas. Tanto nos processos neoplásicos quanto nos proliferativos não neoplásicos, houve maior frequência de afecções cutâneas, possivelmente relacionada à facilidade de acesso e à visualização das lesões.

\section{CONCLUSÃO}

Com base nos dados retrospectivos deste estudo, foi possível determinar as principais características relacionadas à raça, ao sexo e à idade dos felinos domésticos necropsiados na rotina diagnóstica do HV-UFMG em 10 anos do laboratório de patologia. As principais causa mortis em gatos domésticos foram decorrentes de politraumatismo, peritonite infecciosa felina, linfoma e lipidose hepática. A ocorrência de 
traumas foi maior em gatos com até 24 meses. Em relação aos processos neoplásicos e degenerativos, houve predileção por animais idosos. As lesões encontradas à necropsia, juntamente com exames de histopatologia, foram fundamentais para o estabelecimento da causa mortis.

\section{AGRADECIMENTOS}

As atividades de pesquisa no Setor de Patologia recebem suporte financeiro do $\mathrm{CNPq}$ (Conselho Nacional de Desenvolvimento Científico e Tecnológico), da Fapemig (Fundação de Amparo à Pesquisa do Estado de Minas Gerais) e da Capes (Coordenação de Aperfeiçoamento de Pessoal de Nível Superior). RMCG, RS e RLS são bolsistas do CNPq.

\section{REFERÊNCIAS}

CAVE, T.A.; THOMPSON, H.; REID, S.W. et al. Kitten mortality in the United Kingdom: a retrospective analysis of 274 histopathological examinations (1986 to 2000). Vet. Rec., v.151, p.497$501,2002$.

COUTO, S.S.; GRIFFEY, S.M.; DUARTE, P.C.; MADEWELL, B.R. Feline vaccine-associated fibrosarcoma: morphologic distinctions. Vet. Pathol., v.39, p.33-41, 2002.

CCZ-PBH. Centro de Controle de Zoonoses da Prefeitura de Belo Horizonte, 2017. Disponível em: <http://portalpbh.pbh.gov.br/pbh/ecp/comunidade.do? evento $=$ portlet $\&$ pIdPlc $=$ ecpTaxonomiaMenuPortal $\& a$ $\mathrm{pp}=$ saude $\&$ tax $=20618 \&$ lang $=$ pt_BR $\& p g=5571 \& \operatorname{tax}=$ 0\&>. Acessado em: 05 fev. 2018.

DOHOO, I.; MARTIN, W.; STRYHN, H. Logistic Regression. In: DOHOO, I.; MARTIN, W.; STRYHN, H. (Eds.). Veterinary epidemiologic research. Charlottetown: Atlantic Veterinary College, 2003. p.396-398.

FIGHERA, R.A.; GRAÇA, D.L. Sistema hematopoiético. In: SANTOS, R.L.; ALESSI, A.C. (Eds.). Patologia veterinária. Rio de Janeiro: Roca, 2016. p.344-362.

FIGHERA, R.A.; SOUZA, T.M; SILVA, M.C. et al. Causas de morte e razões para eutanásia de cães da Mesorregião do Centro Ocidental Rio-Grandense (1965-2004). Pesqui. Vet. Bras., v.28, p.223-230, 2008.
GUEDES, R.M.C.; BROWN, C.C.; SEQUEIRA, J.L. et al. Sistema digestório. In: SANTOS, R.L.; ALESSI, A.C. (Eds.). Patologia veterinária. Rio de Janeiro: Roca, 2016. p.149-150.

Instituto Brasileiro de Geografia e Estatística (IBGE), 2013. Disponível em: <http://www.ibge.gov.br/home/ estatistica/pesquisas/sintese.php>. Acessado em: 21 jan. 2017.

MARLET, E.F.; MAIORKA, P.C. Retrospective analyzes of cruelty toward dogs and cats in the city of São Paulo. Braz. J. Vet. Res. Anim. Sci., v.47, p.385394, 2010.

MEUTEN, D.J. Tumors in domestic animals. 4.ed. Ames: Iowa State Press, 2002. 800p.

OLSEN, T.F.; ALLEN, A.L. Causes of sudden and unexpected death in cats: a 10-year retrospective study. Can. Vet. J., v.42, p.61-62, 2001.

SCHMIDT, J.M.; NORTH, S.M.; FREEMAN, K.P. Feline paediatric oncology: retrospective assessment of 233 tumours from cats up to one years (1993 to 2008). J. Small Anim. Pract., v.51, p.306-311, 2010.

SCHWEIGERT, A.; CASTANHEIRA, T.L.L.; MACHADO, A.A. et al. Prevalência de neoplasias em gatos domésticos em Araçatuba e região: estudo retrospectivo (1997-2007). Arq. Bras. Med. Vet. Zootec., v.18, p.52-56, 2011.

SIQUEIRA, A. Estudo retrospectivo da casuística em gatos domésticos (Felis catus, Lineu, 1758) do serviço de patologia animal do Departamento de Patologia da FMVZ/USP entre 1998-2008. 2011. 122f. Dissertação. (Mestrado em Ciências) - Faculdade de Medicina Veterinária e Zootecnia, Universidade de São Paulo, São Paulo.

SIQUEIRA, A.; SALVAGNI, F.A.; YOSHIDA, A.S. et al. Poisoning of cats and dogs by the carbamate pesticides aldicarb and carbofuran. Res. Vet. Sci., v.102, p.142-114, 2015.

TOGNI, M.; MASUDA, E.K.; KOMMERS, G.D. et al. Estudo retrospectivo de 207 casos de tumores mamários em gatas. Pesqui. Vet. Bras., v.33, p.353358, 2013.

VOGT, A.H.; RODAN, I.; BROWN, M. et al. AAFPAAHA Feline life stage guidelines. J. Am. Anim. Hosp. Assoc., v.46, p-70-85, 2010.

XAVIER, F.G.; RIGHI, D.A.; SPINOSA, H.S. Toxicologia do praguicida aldicarb ("chumbinho"): aspectos gerais, clínicos e terapêuticos em cães e gatos. Cienc. Rural, v.37, p.1206-1211, 2007. 